\title{
Lectura y escritura en la universidad: contribución para reconstruir una historia
}

\section{Reading and Writing in University: Contribution for Reconstructing a History \\ Lecture et écriture à l'université: contribution pour reconstruire une histoire \\ Leitura e escrita na universidade: contributo para reconstruir uma historia}

Fecha de recepción: 23 DE MARZO DE 2013 / Fecha de aceptación: 6 DE MAYO DE 2015 / Fecha de disponibilidad en línea: 30 DE OCTUBRE DE 2015

Encuentre este artículo en http://magisinvestigacioneducacion.javeriana.edu.co/

(c) (i)

Escrito por Adriana Salazar-Sierra
Pontificia Universidad Javeriana
Bogotá, Colombia
adriana.salazar@javeriana.edu.co
Olga SeVILlA-RenGIFO
Fundación Universitaria MonserRAte
Bogotá, Colombia
calipso314@gmail.com

\author{
Blanca González-Pinzón \\ PontiFIIA UnIVERSIDAD JAVERIANA \\ BogotÁ, COLOMBIA \\ gonzalezb@javeriana.edu.co \\ Carlos Enrique Mendoza-Arciniegas \\ Fundación Universitaria del Área Andina \\ BogotÁ, Colombia \\ cmendoza@areandina.edu.co \\ Alberto Echeverri-Guzmán \\ Universidad Distrital Francisco José de Caldas
Bogotá, Colombia \\ escarabajo4747@gmail.com \\ Daniel Quecán-Castellanos \\ Universidad de Ciencias Aplicadas y Ambientales \\ Bogotá, Colombia \\ dquecanc@udca.edu.co
}

\begin{abstract}
doi:10.11144/Javeriana.m8-16.leuc
\end{abstract}
Luis Ernesto Pardo-Rodríguez UnIVERSIDAD de LA SALLE BogotÁ, CoLombia Ipardo@unisalle.edu.co

María Floralba Angulo-Abaunza UnIVERSIDAD DE LA SALLE BogotÁ, Colombia mafloa29@yahoo.com

Juan Manuel Silva-García Fundación Universitaria del Área Andina BogotÁ, COLOMBIA juanmasilva@hotmail.com

Mariano LozAno-Ramírez Universidad de La SABANA BOGoté, Colombia marianolozanor@gmail.com

\section{Resumen}

Este artículo de investigación presenta los resultados del análisis general de 34 experiencias en lectura y escritura en educación superior en Colombia, realizado a partir de un estudio exploratorio, entre 2008 y 2010, en el marco de la Red de Lectura y Escritura en Educación Superior (REDLEES). Este estudio surge como una necesidad de hacer balance y reconstruir la historia sobre las motivaciones iniciales que movieron a varias universidades colombianas a crear diferentes asignaturas y programas para fortalecer procesos de lectura y escritura de los estudiantes. Como resultado, se confirman la tendencia a diseñar asignaturas con carácter remedial, los esfuerzos individuales y no institucionales y la concepción de la lectura y la escritura como habilidades.

\section{Palabras clave}

Alfabetización; estrategias educativas; enseñanza de la lectura; enseñanza de la escritura; política educacional

\section{Transferencia a la práctica}

Este estudio tuvo una particular incidencia en decisiones investigativas en el marco de la Red de Lectura y Escritura en Educación Superior (REDLEES). A partir de los hallazgos, se tomó la decisión de realizar una investigación entre 2011 y 2012 para caracterizar las prácticas de lectura y escritura de los estudiantes en el nivel anterior a la universidad (en Colombia, educación media) y así comprender la transición de la educación media a la educación superior que experimentan los estudiantes. Además, esta nueva investigación permitió indagar por el concepto de alfabetización académica en los cursos iniciales de lectura y escritura que proponen 12 universidades colombianas.

Para citar este artículo / To cite this article / Pour citer cet article / Para citar este artigo

Salazar-Sierra, A.; Sevilla-Rengifo, O.; González-Pinzón, B.; Mendoza, C.; Echeverri, A.; Quecán, D.; Pardo, E.; Angulo, F.; Silva, J. M. \& Lozano, M. (2015). Lectura y escritura en la universidad: contribución para reconstruir una historia. magis, Revista Internacional de Investigación en Educación, 8(16), 51-70. http://dx.doi.org/10.11144/Javeriana.m8-16.leuc 


\section{Keywords}

Literacy; educational strategies; reading instruction; teaching of writing; educational policy

\section{Abstract}

This article presents the analysis of 34 experiences conducted in Universities in Colombia that involved reading and writing. This analysis was based on an exploratory study conducted between 2008 and 2010 as part of the Literacy Network in Higher Education (Red de Lectura y Escritura en Educación Superior-REDLEES). This study emerges as a necessity to reconstruct the history of the initial motivations that prompted several Colombian universities to propose different courses and programs in order to strengthen students' processes of reading and writing. As a result, the tendency to design courses with remedial nature, individual and non-institutional efforts, and the conception of reading and writing as skills are confirmed.

\section{Transfer to practice}

This study had a particular impact on investigative decisions within the Literacy Network in Higher Education (Red de Lectura y Escritura en Educación Superior -REDLEES). Based on the findings it was decided to conduct a research between 2011 and 2012 to characterize the literacy practices of students in the previous level to university (in Colombian secondary education), in order to understand the transition that students experience from high school to university. In addition, this new research allowed to investigate the concept of academic literacy in the initial literacy courses offered by 12 Colombian universities.

\section{Mots clés}

Alphabétisation; stratégies éducatives; enseignement de la lecture; enseignement de l'écriture; politique d'éducation

\section{Résumé}

Cet article de recherche présente les résultats de l'analyse générale de 34 expériences de lecture et écriture dans l'éducation supérieure en Colombie, on a réalisé à partir d'une étude exploratoire, entre 2008 et 2010, dans le cadre du Réseau de Lecture et Ecriture dans l'Education Supérieure (Red de Lectura y Escritura en Educación Superior) (REDLEES). Cette étude commence en tant qu'un besoin de faire un bilan et reconstruire I'histoire par rapport aux motivations initiales qui ont bougé à plusieurs universités colombiennes à créer les différentes matières d'enseignement et programmes pour renforcer les processus de lecture et écriture des étudiants. En tant que résultats, on a confirmé la tendance à dessiner les matières d'enseignement avec un caractère remédiable, et des efforts individuels et non pas institutionnels, enfin la réception de la lecture et l'écriture en tant qu'habilités.

\section{Transfert à la pratique}

Cette étude a eu une particulaire incidence dans les décisions de la recherche dans le cadre du Réseau de Lecture et Ecriture dans I'Education Supérieure (Red de Lectura y Escritura en Educación Superior) (REDLEES). A partir des trouvailles, on a pris la décision de réaliser une recherche entre 2011 et 2012 pour caractériser les pratiques de lecture et écriture des étudiants dans le niveau antérieur à l'université (en Colombie, Education moyenne educación media) et ainsi comprendre la transition de l'éducation moyenne à l'éducation supérieure qui expérimentent les étudiants. En plus, cette nouvelle recherche q permit d'indaguer par le concept d'alphabétisation académique dans les cours initiales de lecture et écriture qui proposent 12 universités colombiennes.

\section{Palavras-chave}

Alfabetização; estratégias educativas; ensino da leitura; ensino da escrita; política educacional

\section{Resumo}

Este artigo de pesquisa apresenta resultados da análise geral de 34 experiências sobre leitura e escrita em educação superior na Colômbia, realizada a partir de estudo exploratório, entre 2008 e 2010, no marco da Red de Lectura y Escritura en Educación Superior (Rede de Leitura e Escrita de Ensino Superior - REDLEES). Este estudo surge como uma necessidade de fazer balanço $e$ reconstruir a história das motivações iniciais que movimentaram a várias universidades colombianas a criar diferentes matérias e programas para fortalecer processos de leitura e escrita dos discentes. Como resultado, confirma-se a tendência para desenhar matérias de carácter corretivo os esforços individuais, mas não institucionais e a concepção da leitura e a escrita como habilidades.

\section{Transferência à prática}

Este estudo teve particular incidência nas decisões investigativas no contexto da Red de Lectura y Escritura en Educación Superior (REDLEES). A partir dos achados, decidiu-se realizar uma pesquisa entre 2011 e 2012 para caracterizar as práticas de leitura e escrita dos discentes no nível anterior à universidade (na Colômbia, ensino médio) e compreender assim o transito do ensino médio para o ensino superior experimentado pelos discentes. Além, esta nova pesquisa permitiu investigar sobre o conceito de alfabetização académica nas aulas de letramento e literacia propostos em 12 universidades colombianas. 


\section{Introducción}

En Colombia, al igual que en otros países de América Latina, la lectura y la escritura en la educación superior se han convertido en objeto de interés debido a que estos procesos inciden directamente en el desempeño académico de los estudiantes y en temas tan sensibles en la vida de las instituciones como la calidad académica, la producción de conocimiento, las dificultades de comunicación, la repitencia y la deserción, entre otros.

Para discutir precisamente estas problemáticas, desde 2006 en Colombia, se conformó la Red de Lectura y Escritura en Educación Superior (REDLEES), cuya misión fue "Articular los esfuerzos de los directivos, los investigadores y los docentes de las Instituciones de Educación Superior (IES) del país en torno a la lectura y la escritura en dicho contexto" (REDLEES, 2007).

Así, desde el comité de investigación del Nodo Centro de esta Red, se estructuró un estudio exploratorio con el propósito de describir, en primer lugar, el tipo de experiencias iniciales de lectura y escritura en las diferentes universidades del país; en segundo lugar, conocer las motivaciones que, en su momento, llevaron a la creación de tan diversas propuestas y por último, desde el registro de quienes tenían la responsabilidad de proponer, desarrollar y evaluar estos procesos, hacer un balance descriptivo para conocer lo que estaba sucediendo en los currículos de las instituciones con estos procesos y de qué manera eran concebidos. Esto nos permitió caracterizar las experiencias, promover la reflexión y establecer posteriores líneas de acción e investigación.

La historia recogida con este estudio y la subsiguiente compilación de estrategias e investigaciones producidas en las universidades dieron lugar para que entre 2013 y 2014, con participación de diferentes colegas de las universidades que conforman la Red, se produjera el texto Reflexiones y propuestas para pensar una política institucional de desarrollo de la lectura y la escritura en Educación Superior (REDLEES, 2014). El documento tuvo como propósito propiciar la discusión sobre la necesidad de promover en las instituciones programas y estrategias acordes con los retos y necesidades educativas del país en el ámbito de la educación superior.

De este ejercicio de reconocimiento surgió la segunda investigación Formación inicial en lectura y escritura en la universidad. De la educación media al desempeño académico en la educación superior (González \& Salazar-Sierra, 2015) y en cada institución, diversos programas, estrategias e investigaciones de los que da cuenta el documento de reflexión producido entre 2013 y 2014.

\section{Algunos antecedentes y perspectivas de estudio de la lectura y la escritura universitaria en Colombia}

Un punto de referencia importante sobre la dinámica universitaria colombiana en torno al tema de la lectura y la escritura lo proporcionan Blanca Yaneth González-Pinzón y Violetta Vega (2012) al plantear cuatro estadios en este recorrido en las últimas décadas.

En primer lugar, apareció el esfuerzo por ofrecer asignaturas para que los estudiantes de diferentes carreras fortalecieran la comprensión y producción de textos, liderado por la Facultad de Ciencias Humanas de la Universidad Nacional de Colombia en los años 80. Según las autoras, esta es la experiencia de mayor repercusión para la época y no se registran experiencias similares en su momento. Fueron cursos diseñados desde la perspectiva formal de la lengua, similares a los GWSI (General Writing
Descripción del artículo | Article description | Description de l'article | Artigo descrição

Este artículo de investigación se deriva del proyecto Experiencias en lectura y escritura en educación superior, realizado en Bogotá-Colombia por el Comité de Investigaciones - Nodo Centro de la Red de Lectura y Escritura en Educación Superior (ASCUN REDLEES) durante 2009 y 2010. 
Skills Instructions) estadounidenses, populares cien años atrás'.

El segundo momento importante es la promulgación de la Ley 115 de 1994. Esa ley dio relevancia, entre otros aspectos relacionados con la calidad educativa, a los procesos de lectura y escritura en todos los niveles de la escolaridad, lo cual, con el paso del tiempo, obligó a la educación superior a entrar también en la discusión. Como movilizadores importantes de este proceso se identifican la Cátedra UNESCO para la Lectura y la Escritura (liderada desde la Universidad del Valle) y la Red Colombiana para la Transformación de la Formación Docente en Lenguaje.

Durante los primeros años del siglo $\mathrm{XXI}$, según las autoras, se da el tercer estadio en el cual los procesos de lectura y escritura en la universidad cobraron mayor relevancia. El Instituto Colombiano para la Evaluación (ICFES), en un diario de circulación nacional presenta el siguiente resultado "Los universitarios no saben leer ni escribir, dificultades que se observan en la mala redacción y en la poca capacidad de análisis y comprensión de lectura. Además, presentan fallas en la construcción, argumentación, descripción y síntesis de textos" (citado por González \& Vega, 2012, p. 198); situación que confirma la Universidad Nacional, a partir de una investigación realizada desde la División de Admisiones en la que se reportan debilidades en la capacidad analítica, expresiva y argumentativa de los estudiantes que ingresan (Flórez \& Cuervo, 2005, citadas por González, 2012). La perspectiva de este análisis se realizó desde las deficiencias del estudiante sin tener en cuenta factores incidentes en esas manifestaciones. Las Instituciones de Educación Superior (IES) fueron asumiendo poco a poco la responsabilidad, prioritariamente con cursos para los primeros años; como fenómeno interesante, aparecieron las asociaciones para abordar la temática de manera colectiva; una de estas agrupaciones es la apoyada por la Asociación Colombiana de Universidades (ASCUN) con la creación de REDLEES en 2006.

El cuarto estadio es reciente y aún está en consolidación; en este podrían ubicarse todos los esfuerzos de las instituciones por acompañar al estudiante de manera integral — no solo desde la mirada gramatical propia del estadio 1- en la configuración de sus procesos de lectura y escritura de acuerdo con sus necesidades, las cuales en algunos casos superan el ámbito académico. En este período se ubica la gran mayoría de investigaciones sobre el tema; varias de ellas desde colectivos universitarios. El concepto de alfabetización

1 David Russell hace mención a la génesis de estos General Writing Skills Instructions (GWSI). David Russell (1995). Activity Theory and its Implications for Writing Instruction. En Joseph Petraglia (ed.). Reconceiving Writing, Rethinking Writing Instruction, 51-78. Hillsdale, New Jersey: Erlbaum. académica gana su lugar, pero también entra en análisis, pues parece ser insuficiente a la hora de pensar en las múltiples movilizaciones de la lectura y la escritura en la complejidad universitaria.

Tomando como base este panorama, los resultados de esta investigación se sitúan en el cuarto estadio. Los hallazgos aquí realizados, sumados a las ya múltiples investigaciones terminadas y en curso sirven de insumo para comprender cómo se produjo esta transformación y revelar si las experiencias en las universidades han sido fuente de cambio, de perpetuación de viejas concepciones o si están en transición.

\section{Metodología del estudio²}

Con el propósito de describir el tipo de experiencias con la lectura y la escritura que se desarrollaron en las IES que conforman la REDLEES, se optó por un estudio cualitativo de carácter exploratorio.

Al iniciar la investigación (2008), 72 universidades pertenecían a la red, entre ellas, varias públicas y una gran mayoría de privadas, lo que mostraba una directa relación con la realidad nacional, en términos de la naturaleza de nuestras instituciones. Entre octubre de 2009 y mayo de 2010, después de varias convocatorias por correo electrónico, se registraron 34 experiencias -algunas aportadas por los mismos investigadores-, provenientes de 18 universidades de diferentes partes de Colombia (13 de Bogotá, 2 de Santander, 1 de Santa Marta, 1 de Cali y 1 de Popayán).

Para este estudio, se entendió como "experiencia" aquella práctica en el contexto universitario vinculada con la enseñanza y el aprendizaje de la lectura y la escritura, que hubiese demostrado una aproximación destacada y sistemática a la problemática, que se tradujese en la intención de cualificación de los procesos de comprensión y producción textual propios de la cultura universitaria. No se asumió la clasificación de Experiencias como Significativas propuesta por la ONU (citada por MEN, 2006), entendidas como aquellas prácticas que tengan "un impacto demostrable y tangible en la calidad de vida de las personas", porque se pretendió reconocer la existencia de prácticas, entender sus dinámicas, determinar las tendencias; y no, evaluar su calidad.

2 Los investigadores que hicieron parte de este proceso fueron Adriana Salazar, Pontificia Universidad Javeriana, Bogotá; Alberto Echeverri, Universidad Distrital Francisco José de Caldas, Bogotá; Carlos Mendoza y Juan M. Silva, Fundación Universitaria del Área Andina, Bogotá; Daniel Quecán, Universidad de Ciencias Aplicadas y Ambientales, UDCA, Bogotá; Ernesto Londoño y Patricia Carreño, Universidad de San Buenaventura, Bogotá; Floralba Angulo y Ernesto Pardo, Universidad de La Salle, Bogotá; Mariano Lozano, Universidad de La Sabana, Bogotá; Marisol Moreno y Patricia Rey، Universidad Nacional de Colombia, Bogotá; Olga Sevilla, Fundación Universitaria Monserrate, Bogotá; Rafael Ayala, Fundación Universitaria Los Libertadores, Bogotá. 
Para esta investigación, las experiencias las reportaron sus responsables, generalmente profesores de planta. Como estudio exploratorio, dado que era la primera aproximación sistemática dentro de la REDLEES a las prácticas de lectura y escritura de las universidades que la conformaban, se consideró un punto de partida parcial, pero adecuado como balance para dejar registro de este fenómeno en el país. Iniciar con un reconocimiento desde los integrantes de la REDLEES sobre nuestras prácticas nos permitió encontrar puntos en común, diferencias y problemáticas. Se convirtió en una oportunidad para investigaciones puntuales futuras.

Para recoger la información, el equipo investigador construyó un cuestionario de 16 preguntas, que podía contestarse en línea o en un archivo de Excel para ser enviado por correo electrónico. La mayoría prefirió esta última posibilidad. Al tratarse de un estudio exploratorio, las preguntas intentaban obtener información sobre los rasgos generales de una experiencia de enseñanza y aprendizaje de la lectura y la escritura en el contexto universitario. Este cuestionario fue de creación colectiva y se discutió en las reuniones del Nodo Centro de la Red. Desde esta óptica, el equipo investigador definió cuatro grandes aspectos: los datos generales de identificación de la experiencia, su descripción, un balance de la implementación y las proyecciones de continuidad de la experiencia.

En cuanto a los datos generales, resultó importante identificar el nombre y tipo de experiencia (investigación, curso obligatorio, curso electivo, prueba, asesoría individual, asesoría colectiva, actividades, otros), la institución, los responsables, las palabras clave en las cuales se enmarcaba y el resumen. La descripción de la experiencia se concentró en las problemáticas que dieron lugar a su origen y los aspectos pedagógicos involucrados. En esta resultaron relevantes el objetivo de la experiencia, los destinatarios, los antecedentes y la trayectoria, el enfoque conceptual y los aspectos metodológicos considerados. En el balance de la experiencia, se pretendió describir sus logros y sus retos, y los mecanismos de socialización. Por último, con relación a las proyecciones de la experiencia, se indagó por la posibilidad de transferencia y los mecanismos de financiación.

En síntesis, el cuestionario quedó conformado por 16 puntos que dieron cuenta de cada experiencia, así:

Tabla 1

Cuestionario

\begin{tabular}{|c|c|}
\hline $\begin{array}{l}\text { Datos } \\
\text { generales }\end{array}$ & $\begin{array}{l}\text { 1. Nombre y tipo de experiencia } \\
\text { 2. Institución } \\
\text { 3. Responsables (nombre, cargo, tipo de vinculación, correo electrónico) } \\
\text { 4. Palabras clave (cinco) } \\
\text { 5. Resumen }\end{array}$ \\
\hline $\begin{array}{l}\text { Descripción } \\
\text { de la } \\
\text { experiencia }\end{array}$ & $\begin{array}{l}\text { 6. Objetivo } \\
\text { 7. Destinatarios } \\
\text { 8. Antecedentes y motivaciones para su creación } \\
\text { 9. Enfoque conceptual (conceptos y autores representativos que sustentan la experiencia) } \\
\text { 10. Estrategias didácticas } \\
\text { 11. Trayectoria (tiempo durante el cual se ha llevado a cabo la experiencia y la frecuencia de aplicación de la misma) }\end{array}$ \\
\hline Balance & $\begin{array}{l}\text { 12. Logros } \\
\text { 13. Retos } \\
\text { 14. Socialización (productos que hacen públicos los conocimientos relacionados con la experiencia de lectura o } \\
\text { escritura y su incidencia en la apropiación social) }\end{array}$ \\
\hline Proyecciones & $\begin{array}{l}\text { 15. Posibilidades de transferencia (en qué condiciones considera que sería posible la transferencia de la } \\
\text { experiencia a otras instituciones) } \\
\text { 16. Financiación (cómo se ha financiado la experiencia) }\end{array}$ \\
\hline
\end{tabular}

Fuente: elaboración propia 
Para el análisis, se tuvieron en cuenta las tendencias en cada uno de los puntos registrados en las experiencias. En los aspectos en los que fue posible agrupar datos numéricos, se tomó como referencia la media. En los aspectos cualitativos se agruparon tendencias, tomando como categorías de base las del formulario. A continuación, se presentarán los hallazgos más significativos que, a su vez, están sistematizados como datos estadísticos por Mendoza et al. (2011).

\section{¿Qué relevaron los datos generales aportados por las experiencias?}

En el primer grupo, el de los datos generales, es de señalar la alta participación en este estudio de instituciones de carácter privado, y entre ellas algunas de carácter confesional definido, en coherencia con la evolución histórica de la universidad colombiana. Los proyectos curriculares o las facultades de las que provenían las experiencias y a las que servían son muy diversos: en ocasiones, se trataba de instancias ofrecidas a toda la población estudiantil de la institución; en otros estaban dirigidas a estudiantes de una sola carrera o de varias dentro de una facultad, a toda la facultad o a programas de diferentes facultades. Algunas experiencias registradas (12) eran investigaciones o se derivaban de estas.

Predominaba la diversidad; cada institución respondía de manera particular a las necesidades de comprensión y producción textual; sin embargo, en su mayoría, estas respuestas aparecían como esfuerzos particulares de grupos de profesores generalmente de planta. Pocas experiencias tenían un carácter derivado de una política institucional (2) y si lo tenían, llevaban pocos años. Se observó una oportunidad: los esfuerzos aislados podrían consolidarse para así contribuir a su cualificación, si se entraba en franco diálogo con los directivos y comenzaban a promoverse movilizaciones que involucraran a más docentes de diversos campos del saber.

En cuanto al contenido de las propuestas, las palabras clave ofrecían pistas de su orientación. Había predominio de la "comprensión" (7 veces) y del uso de "estrategias" para hablar de lo que se hacía (9 veces). Esto era evidencia de una tendencia en los resultados del estudio: la preocupación por los procesos de lectura y la forma didáctica de abordarlos. No apareció con igual relevancia la palabra "escritura", a pesar de que la discusión sobre escritura en las disciplinas ya había estado presente en diferentes eventos académicos en el país, pero aún no era un interés manifiesto, al menos en las experiencias estudiadas.

En directa relación con lo anterior, resultaba interesante cómo las experiencias enfatizaban en: lectura $21 \%$, escritura $15 \%$, lectura y escritura $52 \%$, lectura, escritura y oralidad $12 \%$. Se observó que la mayoría de las propuestas integraba los componentes de lectura y escritura, pero, cuando se analizaron en profundidad, predominaba la lectura. Surgieron pues dos interrogantes ipor qué la escritura era atendida en menor proporción que la lectura?, ¿por qué algunas propuestas integraban la oralidad?, quizá esta última pregunta tuviera relación con la tendencia al trabajo de estos temas con el enfoque de las habilidades comunicativas.

Por otra parte, el tipo de experiencias registradas fue diverso:

Tabla 2

Tipo de experiencia

\begin{tabular}{|c|c|c|c|c|c|c|c|c|}
\hline $\begin{array}{l}\text { Criterio } \\
\text { Tipo de } \\
\text { experiencia }\end{array}$ & Investigación & $\begin{array}{l}\text { Prueba } \\
\text { diagnóstica }\end{array}$ & $\begin{array}{l}\text { Concurso } \\
\text { - material } \\
\text { apoyo }\end{array}$ & $\begin{array}{l}\text { Actividades } \\
\text { talleres }\end{array}$ & $\begin{array}{l}\text { Curso } \\
\text { obligatorio }\end{array}$ & $\begin{array}{l}\text { Curso } \\
\text { electivo }\end{array}$ & $\begin{array}{l}\text { Asesoría } \\
\text { individual }\end{array}$ & $\begin{array}{l}\text { Asesoría } \\
\text { colectiva }\end{array}$ \\
\hline Porcentaje & $32,35 \%$ & $11,76 \%$ & $17,64 \%$ & $8,82 \%$ & $15,70 \%$ & $5,88 \%$ & $2,94 \%$ & $2,94 \%$ \\
\hline Frecuencia & 12 & 4 & 6 & 3 & 5 & 2 & 1 & 1 \\
\hline Componentes & \multicolumn{2}{|c|}{$\begin{array}{c}44,11 \% \\
\text { Vinculados con ejercicios } \\
\text { de investigación }\end{array}$} & \multicolumn{2}{|c|}{$\begin{array}{c}26,46 \% \\
\text { Hacen parte de las } \\
\text { propuestas de extensión } \\
\text { de la universidad o son } \\
\text { cursos libres }\end{array}$} & \multicolumn{4}{|c|}{$\begin{array}{l}27,46 \% \\
\text { Experiencias propias de aula. Docencia }\end{array}$} \\
\hline
\end{tabular}

Fuente: elaboración propia, a partir de la agrupación del tipo de experiencia 
Es interesante la presencia de los tres componentes propios de la educación superior: la investigación, la extensión y la docencia para abordar la lectura y la escritura en la universidad. Aunque la interrelación entre estos tres aspectos no era tan clara ¿qué tanto impactaban los procesos de investigación la práctica docente?; ¿de dónde provenían las experiencias de extensión? y iconstituía la actividad docente un escenario de investigación? Llamó la atención cómo la mayoría de las experiencias surgió por fuera de políticas institucionales y se propuso al margen de los planes de estudio; un grupo de ellas (9) respondió a las necesidades de proyección de la universidad y otras (12) eran producto de procesos de investigación.

\section{Descripción de las experiencias}

¿Cuál fue el propósito predominante de las experiencias?

El propósito de las experiencias — dato aportado por uno de los criterios del segundo aspecto- fue, predominantemente (80\%), "desarrollar", "mejorar" y "caracterizar" aspectos relacionados con la comprensión y producción textual. Esto coincidía con una perspectiva más de carácter remedial, en la que se desconocía que el ingreso a la universidad implicaba nuevos aprendizajes vinculados con la lectura y la escritura en contextos particulares de las disciplinas. Esto, a pesar de que en el país ya había ingresado la discusión de la necesidad de situar estos procesos de enseñanza aprendizaje como parte del estudio en los diferentes campos del saber (Bazerman, 1988; Bazerman, Little, Chavkin, Fouquette, Bethel \& Garufis, 2005; Bazerman \& Prior, 2005; Bazerman \& Russell, 1995; Carlino, 2009, 2005; Greene \& Lidinsky, 2008).

¿Para quiénes se ofrecian las experiencias sobre lectura y escritura?

La mayor parte de las experiencias iba dirigida a estudiantes de pregrado (52\% a los de I a III semestre; $48 \%$ a los de IV y posteriores). A los de pregrado y posgrado, $9 \%$ y exclusivamente a los de posgrado, 3\%. A estudiantes, docentes y administrativos, $6 \%$. A estudiantes y profesores, $6 \%$.

El mayor porcentaje de las experiencias dirigidas a pregrado se vinculó con el componente de investigación (51,72\%). Iguales porcentajes $(24,13 \%)$ se observaron en las de docencia y extensión. Ninguna de las de extensión superaba el ámbito estricto de los pregrados y de las de investigación, el $3 \%$ se dedicaba a la vez a pregrado y a posgrado. Solo una experiencia se ocupaba del nivel de maestría, a pesar de que la mirada general de muchos docentes de las maestrías y doctorados en Colombia constataba ya las necesidades de los desarrollos escriturales y lectores en ese nivel universitario.

Vale la pena mencionar que a pesar de ir dirigidas a diferentes niveles y necesidades, terminaron siendo similares en estrategias y propósitos, lo que anticipó una concepción de la lectura y la escritura como competencias generales, libres de dominio.

¿Por qué surgieron las experiencias y cómo se justificaba su creación?

En cuanto a los problemas que justificaban la realización de las experiencias generadas como propuestas de investigación, la mayor tendencia estaba relacionada con la identificación de las dificultades que manifestaron los estudiantes con la lectura y la escritura que el contexto de la educación superior exigía: análisis, interpretación, argumentación, desarrollo de pensamiento lógico y crítico. En segundo lugar, llama la atención la responsabilidad que se les asignó a los métodos tradicionales de enseñanza como causa de la problemática. 
La coincidencia de estas valoraciones con lo expuesto en los objetivos de la mayoría de las propuestas (27), expresados con verbos como fortalecer, mejorar, desarrollar, enseñar, etc., hacía visible la preocupación por darle al estudiante aquello con lo que no contaba que, además, en varias de las experiencias (5) se atribuía expresamente a los niveles educativos anteriores.

De otra parte, se destacaban tres aspectos esenciales, por su escasa mención en los datos ofrecidos: en primer lugar, la incidencia del rol de los docentes como modelos de lectura y escritura (solo dos experiencias lo mencionan); en segundo lugar, la interacción de los llamados "nativos digitales" con la lectura y la escritura en los medios electrónicos (dos experiencias lo mencionan) y, en tercer lugar, la necesaria ubicación de estos procesos como ejercicios transversales a todas las asignaturas, condición de la cultura académica (dos experiencias lo mencionan).

\section{Consideraciones sobre teorías, modelos y marco conceptual}

En la tabla 3 se sintetizan los autores más mencionados, de acuerdo con el aspecto Descripción de la experiencia, que hizo parte de los criterios iniciales de recolección y análisis:

Tabla 3

Autores más mencionados

\begin{tabular}{l|l|l|l|l|l|l}
\hline $\begin{array}{l}\text { Daniel } \\
\text { Cassany }\end{array}$ & Paula Carlino & $\begin{array}{l}\text { Lev } \\
\text { Semiónovich } \\
\text { Vigotsky }\end{array}$ & $\begin{array}{l}\text { Miguel de } \\
\text { Zubiría }\end{array}$ & $\begin{array}{l}\text { Teun A. van } \\
\text { Dijk }\end{array}$ & $\begin{array}{l}\text { Roland } \\
\text { Barthes }\end{array}$ & $\begin{array}{l}\text { Jürgen } \\
\text { Habermas }\end{array}$ \\
\hline $22,85 \%$ & $20,00 \%$ & $14,28 \%$ & $14,28 \%$ & $11,42 \%$ & $8,75 \%$ & $8,75 \%$ \\
\hline
\end{tabular}

Fuente: elaboración propia

Se observa en muchos casos eclecticismo. Llama la atención la dificultad que representó para los respectivos reportes precisar el tipo de enfoque de los cursos o las investigaciones; se nombraban autores y libros, pero en la mayoría de los casos no se construía una argumentación para un enfoque definido y sin hacer referencia a una descripción de sus particulares teorías. Mientras Daniel Cassany y Paula Carlino tenían la prioridad para los casos de extensión, Daniel Cassany, Paula Carlino, Lev Vigotsky y Miguel de Zubiría aparecían en lo referente a investigación. Vigotsky llevaba la delantera en docencia, seguido de Daniel Cassany y Roland Barthes. Así como se consideraban competencias generales, se puede decir que al quehacer se le integraban diversas visiones, sin importar que entre ellas hubiese posiciones en ocasiones encontradas y disímiles. Esto estaba en desencuentro con lo que se vería sobre la didáctica, en la que predominaba el taller, que tendía a llevarse a cabo desde prácticas canónicas de comprensión y orientado hacia aspectos formales de lengua; en total oposición con lo que los autores mencionados plantean.

Una explicación que arriesgamos en su momento con este estudio es que al tratarse de un campo en exploración y paulatina consolidación, era natural que se probara desde diferentes miradas y autores, pues para la época no se podía hablar de experticia en lectura y escritura universitarias. Los estados del arte que circulan actualmente en el país sobre el tema, reconfirman la diversidad conceptual con la que se han estudiado estos procesos (Ortiz, 2011; Pérez \& Rincón, 2013; Uribe \& Camargo, 2011). 


\section{A propósito de las estrategias didácticas visibles}

El siguiente desglose porcentual muestra cuál estrategia didáctica predominó o tuvo mayor presencia en las diferentes experiencias. Esto hace manifiesto que los ejercicios en el aula (35\%) ocuparon la mayor parte de la atención de docentes y estudiantes. El porcentaje siguiente (26\%) es de diversa índole didáctica. Relativamente significativas son las estrategias cognitivas (12\%), aunque el enfoque claramente privilegiado, según el apartado precedente, parece ser el cognitivo (y su derivado, el metacognitivo). Las propias del enfoque crítico estaban reportadas en una sola experiencia.

Abundaban pues los recursos didácticos. La revisión de textos tenía su nicho propio, aunque pequeño (15\%), pero más bajo aún era el de los cuentos (6\%). Por su parte, el uso de recursos electrónicos, propio de los desempeños informáticos y comunicativos, apenas si se relevaba (6\%).

Aunque la mayoría de experiencias pertenecía al plan obligatorio de estudios, un 1,7\% de ellas mencionó explícitamente que se desarrollaron como asignaturas optativas o electivas, lo que mostró que eran experiencias extracurriculares a las que se asistía libremente. Otro dato interesante es ver cómo tres de estas experiencias realizaban concursos de lectura y escritura para grupos o institucionales, lo que significaba la posibilidad de hacer públicas las competencias relacionadas con las prácticas de lectura y escritura y la necesidad de mostrarlas como importantes.

En cuanto a la lectura, la escritura y la relación con los proyectos de investigación (no en curso, sino como preceptiva para orientarlos como formación), tres experiencias dedicaron sus estrategias didácticas a la planeación y escritura de textos relacionados con sustentaciones, proyectos de investigación formativa y proyectos de investigación en maestría.

Atendiendo a la idea de que las experiencias de investigación son el principio de nuevas problematizaciones sobre el objeto de estudio, surgieron nuevas preguntas: ¿Cuál es la concepción de enseñanza cuando se habla de estrategias didácticas de lectura y escritura?, ¿los métodos en la enseñanza de la lectura y escritura son suficientes para comprender las implicaciones de estas prácticas en la cultura académica? Si no se parte de prácticas reales de lectura y escritura universitaria, ¿no se corre el riesgo de didactizar la lectura y la escritura como prácticas artificiales?

\section{Trayectoria de las experiencias}

El $50 \%$ de las experiencias analizadas (17) se había iniciado recientemente. La convocatoria de participación en REDLEES parecía haber movilizado a las instituciones, directivos y profesores para pensar espacios diversos de promoción de la lectura y la escritura. Solo una experiencia se reportó como terminada, mientras el 96,9\% de los informantes consideró los procesos de lectura y escritura como estrategias de formación permanente que no debían concluir sino retroalimentarse día tras día.

Llama la atención que el índice mayor de experiencias estaba entre los 3 y 5 años, el siguiente correspondía a las que se movían entre los 1 y 3 años, y el menor era el de las que llevaban más de 6 años. Una de extensión había durado 12 años, por supuesto con diversos grupos. Solo en una de las IES la experiencia de investigación había superado los 5 años.

Por supuesto, no en todos los casos la experiencia se refería a un mismo grupo de estudiantes sino a la institucionalización de la experiencia y, por tanto, con diversos grupos, lo que explicaría la falta de continuidad en los procesos individuales. 


\section{Las experiencias desde los logros y los retos}

Gracias a la intención de los docentes de dar a conocer las experiencias que se habían ido construyendo con el tiempo en las diferentes instituciones y las formas de enseñanza, el estudio recogió un análisis de los logros, retos y posibilidades que cada una de ellas aportó para la discusión.

Logros

En este apartado se mencionarán los tipos de logros, de acuerdo con los aspectos universitarios con los que se asocian: lo académico, la investigación, la extensión universitaria, la estructura institucional y la eficacia de las propuestas.

Las experiencias registraron los logros académicos como los más significativos (54,29\%), a pesar de que no registraron los mecanismos de medición; en detalle, los docentes se referían a ellos cuando percibían en los estudiantes mejoramiento en el desarrollo de las competencias, el aumento de la motivación y la concientización sobre la importancia de la lectura y la escritura, el mejor desempeño en la comprensión y la producción textual, el desarrollo de habilidades y capacidades lectoras y escritoras, la participación activa en las clases, la producción de artículos, la apropiación conceptual y el análisis crítico que les permite la lectura y la escritura y el cambio de esquema previo o mentalidad sobre estos dos procesos.

Los logros referidos al desarrollo de la investigación y la producción intelectual se presentaron con un 17,14\%. En este porcentaje se señalaron aspectos como la producción de artículos, la publicación en revistas en medio físico o electrónicas, la creación de semilleros de escritores e investigadores, el diseño de pruebas en lectura y escritura y la concientización de la importancia de la divulgación del conocimiento científico.

Los logros orientados a la relación de la experiencia con el sector externo correspondieron al 17,14\% y tenían qué ver con la participación de la comunidad académica, la implementación de los aprendizajes de la experiencia en otros contextos, los ejercicios de interdisciplinariedad que se habían llevado a cabo, los reconocimientos que había ganado la experiencia dentro de la institución, la revaloración del concepto de lectura y escritura, la participación y organización de concursos de cuento y poesía y la incidencia de la experiencia en la práctica social.

En menor porcentaje, se encontraron los logros referidos a la estructura institucional $(5,71 \%)$, referenciados por el incremento de cobertura poblacional, el reconocimiento institucional de la lectura y la escritura en la cultura académica, la creación de un departamento dedicado a estos procesos y la necesidad de asignar espacios en el currículo; y los logros concernientes a acciones e instrumentos con un 5,71\%, que se manifestaron con la organización de eventos relacionados con la lectura y la escritura, las actividades de reflexión sobre el tema, la creación de módulos virtuales, el diseño de páginas web y la escritura de diversos tipos de documentos académicos.

Con respecto a la eficacia de las propuestas, los logros se agruparon en: 1) el desempeño de los estudiantes frente a la experiencia, 2) los espacios, las acciones o propuestas para la enseñanza de la lectura y escritura y 3) la efectividad de las estrategias en el aprendizaje.

En cuanto al desempeño, los docentes que orientaban las experiencias reconocieron que sus estudiantes mejoraban los niveles de competencia en lectura y escritura, además, se refirieron a las capacidades y habilidades que desarrollaron los estudiantes. Esto inferido desde el discurso con el que se manifestaban los logros obtenidos, mas no como una reflexión clara sobre el enfoque de enseñanza. 
Los espacios, las acciones o la propuesta en sí se consideraron un logro por parte de quienes registraron la experiencia porque generaron conciencia sobre el valor de los procesos de interpretación y comprensión. Por otra parte, se notó un aprendizaje inmediato y aunque no se sabía si iba a haber algún tipo de transposición del aprendizaje en otros contextos, los docentes sintieron que los estudiantes habían aprendido.

A pesar de la soledad de la preparación, diseño y desarrollo de la experiencia, se logró la construcción de módulos de formación, el diseño de espacios virtuales y el manejo de documentación y pruebas específicas. En los casos en los que estos recursos se dieron a conocer, fue posible visibilizar el trabajo realizado; en el caso contrario, era un aporte profesional y didáctico para quien asumía la experiencia como espacio de cualificación de la práctica docente.

La efectividad entendida como logro de la experiencia, estaba dada por el desarrollo de procesos de investigación y por la producción intelectual (artículos con fines de publicación, creación de nuevas revistas, conformación de semilleros de investigación, desarrollo de pruebas diagnósticas, producción de textos literarios), lo que permitía la divulgación de la naciente reflexión en torno a la lectura y la escritura como referente de la cultura académica. La efectividad también era pensada desde la relación con el sector externo de la comunidad académica local, que mediante eventos internos y externos permitían la implementación de nuevos modelos teóricos, el diálogo interdisciplinar, el reconocimiento de pares, la reflexión y, en general, la práctica social de la lectura y la escritura.

\section{Retos}

La información ofrecida por los docentes sobre las experiencias, permitió agrupar los retos en relación con los aspectos académicos y administrativos. Es importante aclarar que la pregunta que se hizo era directamente sobre las dificultades presentadas en la realización de la experiencia, solo que para efectos de proyectar el estudio se propuso convertir esas dificultades en oportunidades.

En cuanto a lo administrativo, con un $36,17 \%$, el tiempo resultó un reto; el tiempo no solo de dedicación por los estudiantes o del asignado a los profesores, en algunos casos se trataba de la superposición de horarios, en otro momento del fraccionamiento del horario entre lo teórico y lo práctico de la experiencia y la necesidad de darle continuidad a la experiencia para evidenciar sus logros e impacto. Por otra parte, el tiempo también fue visto como la posibilidad de un trabajo en el que hubiese participación de otros colegas y las intenciones de la experiencia se trasladasen a la cualificación de la cultura académica en otras disciplinas.
Otro aspecto administrativo reportado como debilidad $(29,78 \%)$, tiene relación con la estabilidad del docente y/o de los grupos de estudiantes, la gestión, la falta de disponibilidad de recursos suficientes, la resistencia al cambio, las dificultades que genera el sistema evaluativo por productos y no por procesos, los pocos espacios de formación dedicados a la lectura y la escritura, el aislamiento y la falta de compromiso de docentes y estudiantes por lo que la experiencia promueve.

Con esta información se pudo inferir que la dificultad que se manifestó con mayor frecuencia en el estudio fue la falta de tiempo y de respeto por el mismo para pensar la formación en lectura y escritura, tiempo que se dedica a la planeación, desarrollo y sobre todo evaluación de los procesos. Además, el tiempo para pensar la lectura y la escritura en el contexto académico de la universidad es insuficiente $y$, a veces, nulo, según las descripciones de las experiencias, condición que promueve un trabajo en solitario con contratos específicos sin una perspectiva impactante en la cultura académica deseada.

Se evidenció, además, una inestabilidad laboral o rotación de los encargados del proceso. Las visiones y los procedimientos de muchos de quienes gestionan la educación superior no son tan ágiles y prospectivos como se quisiera. Se presiona la demostración de resultados y productos, pero fallan la comunicación, la disponibilidad al cambio y la flexibilidad en los mecanismos evaluativos. En consecuencia, los objetivos no son evaluados y la experiencia no es cualificada porque su existencia depende en gran medida de los sujetos que la desarrollan, más aún cuando la experiencia en lectura y escritura no está pensada desde un enfoque curricular o académico, que mantenga su orientación con la presencia o no del docente, para que a largo plazo, pueda hacerse investigación sobre ella.

Por otra parte, en lo académico, desde ya aparecían concepciones e imaginarios acerca de la escritura y la lectura como elementos frágiles dentro del currículo, algo que pertenece más al hacer que al saber. No hay postura institucional en cuanto a cómo estructurarlas y potenciarlas según las modalidades de estudio (presencial, a distancia, o virtual), por lo que el acompañamiento al estudiante es débil y los docentes no siempre cuentan con las competencias para mediar el conocimiento, evaluarlo y resignificarlo. En consecuencia, la falta de fundamentación y acercamiento a una postura conceptual sobre lectura y escritura provocó el desarrollo de la enseñanza de estas prácticas de manera diversa, con enfoques que en muchas ocasiones se contradicen o que no son coherentes con la necesidad y realidad de la cultura académica de la institución de educación superior.

Además, las prácticas aisladas y las estrategias parciales, discontinuas y limitadas en el tiempo, como 
lo muestra el estudio, agotan la didáctica a acciones puntuales, con resultados inmediatos, sin vida dentro de la cultura académica. El reto está en la consolidación de un saber didáctico, que permita que el docente universitario en el espacio curricular que lidera, articule de manera natural las concepciones de la lectura y la escritura académica, con la reflexión sobre la enseñanza de su saber y el contexto académico en el que se encuentran sus estudiantes, para — de cierta manera- llegar a una configuración didáctica que hagan de la lectura y la escritura prácticas de conocimiento, de comunicación y de identidad profesional. Una propuesta que — referida desde el enfoque didáctico del contenido (Acevedo, 2009) - permite la relación de un conocimiento disciplinar con una acción de enseñanza útil para la formación académica.

Otros retos apremiantes se relacionaron con la transferencia del conocimiento, el acompañamiento a la lectura y la escritura desde los procesos personales, las concepciones o imaginarios con los que se llega a la experiencia, la evaluación como producto y no como proceso, la falta de fundamentación clara desde la institución, la modalidad (diurna, nocturna, a distancia) del programa, la relación compleja con la formación en lectura y escritura en los niveles anteriores de formación del estudiante ¿cuál es la responsabilidad de la educación media y cuál la de la universidad?

En síntesis, los retos podían visualizarse en la necesidad apremiante de que la universidad tomara decisiones institucionales sobre la lectura y la escritura, desde la comprensión de la cultura académica que la identifica; esto, como se ha insistido en diversos estudios, puede realizarse mediante la definición de políticas, directrices, orientaciones curriculares, entre otras herramientas. El asunto de la lectura y la escritura debe pensarse desde las prácticas socioculturales, la gestión, el desarrollo de los estudiantes, de los docentes, del currículo, la investigación, la proyección social, en fin, de todos los componentes que hacen parte del ambiente universitario.

\section{Discusión}

La tendencia a atribuir las dificultades de lectura y escritura de los estudiantes a los niveles de educación anterior ya está ampliamente cuestionada, pero impera aún. Esto merece una reflexión frente a dos temas en principio: los tipos de textos a los que se enfrentan los estudiantes en estos espacios y las formas de evaluación mediante las cuales se valora su interacción con los textos. En este sentido, la profesora Emilia Ferreiro (2002) aclara que la alfabetización es un continuo que va de la infancia a la edad adulta $y$, dentro de la edad adulta, un continuo de desafíos cada vez que nos enfrentamos con un tipo de texto con el cual no hemos tenido experiencia previa. En consonancia con esta idea, Luis Bernardo Peña-Borrero (2009) y Paula Carlino $(2009,2005)$ claramente establecen una diferencia entre leer en la secundaria y leer en la universidad; en la universidad, el estudiante se enfrenta a textos y exigencias de lectura y escritura completamente nuevos para él. Así, el estudio invita a profundizar en el tema para analizar las interacciones de los estudiantes en los espacios propios de sus carreras y las formas de leer y de escribir a las que se someten en cada uno de ellos.

En términos de la evaluación, también es importante analizar cómo muchas de las dificultades se nombran de manera genérica como "problemas con la comprensión y la interpretación", con base en muchos de los casos en test de selección múltiple o ejercicios para rendir cuentas sobre lo que dice un texto, sin observar, como se ha dicho, las múltiples interacciones de los sujetos con los textos. Blanca Yaneth GonzálezPinzón y Violetta Vega (2010) a este respecto afirman:

\begin{abstract}
Varias estrategias didácticas de orientación en este campo se denominan "ejercicios de comprensión de lectura" y muchos de los espacios destinados a su estudio "talleres de comprensión de lectura". Pero, ¿qué es lo cuestionable en estas situaciones? Que la comprensión es solo un aspecto del proceso, no el fin último. Por ello, el interés por que en la universidad se aclaren los objetivos de la lectura. Después de hacer la comprensión, el estudiante debe saber qué hacer con esa información que ya entendió. Sería más claro hablar, por ejemplo, de lectura para la investigación, lectura para organizar información, lectura para resolver un problema, lectura para el aprendizaje conceptual, lectura para comparar teorías o puntos de vista, lectura para refutar, lectura para hacer un comentario, etc. (p. 111).
\end{abstract}

Lo que este estudio refleja responde claramente al proceso que ha llevado la discusión sobre la lectura y la escritura en educación superior en Colombia. Hasta hace una década, no se discutían a profundidad sus necesidades, sus características, ni las prácticas llevadas a cabo en los espacios académicos. Era muy poca la literatura que circulaba a este respecto, y las acciones adelantadas para planear programas y estrategias en pro de su desarrollo partieron de concepciones, creencias y conocimientos aportados por docentes de las áreas de la literatura, la lingüística, la comunicación y las ciencias humanas en general. Así mismo, aunque no en todos los casos, se asimilaron los procesos de afianzamiento en la educación superior a los procesos llevados a cabo en niveles educativos anteriores y, como se ha dicho, con un carácter remedial. A la fecha, ya circulan varias publicaciones en las que 
se hacen aproximaciones al estado de la discusión (Camargo, Uribe, Caro \& Castrillón, 2009; Cisneros \& Vega, 2011; González, 2009; González \& Vega, 2010, 2012; Pérez et al., 2010) y que no revelan una corriente clara, sino variados enfoques que, se podría decir, están a prueba.

De acuerdo con Blanca Yaneth González-Pinzón y Violetta Vega (2012):

Las 125 investigaciones registradas [en Colciencias] que tratan el tema de la lectura y la escritura universitaria se dedican a la validación de estrategias didácticas, al estudio de concepciones y prácticas de lectura y escritura, al establecimiento de las capacidades de los estudiantes en estos temas y a la exploración de herramientas virtuales que contribuyan al mejoramiento de los procesos de formación. En dichas experiencias priman como enfoques la psicología cognitiva y el constructivismo y en la bibliografía de soporte se repiten autores como Cassany, Vigotsky, Piaget, Van Dijk y los colombianos Martínez, M. C. y De Zubiría. Como puede verse, son muy pocas las indagaciones que se concentran en analizar el tipo de práctica que efectivamente se lleva a cabo en las cátedras. Aunque la mayoría de las propuestas tienen como propósito mejorar y cubrir dificultades, todo esto revela cierto hermetismo ante las discusiones actuales en las prácticas docentes y un escaso interés en otorgar nuevos sentidos a los conceptos lectura y escritura.

... debe destacarse que la discusión sobre lectura y escritura académica en Colombia se ha transformado: la óptica ineludible de la lingüística y la concepción de que el estudiante es el absoluto responsable de su proceso de escritura en la universidad han abandonado el centro de la escena. Con todo, aún es necesario consolidar políticas institucionales que favorezcan el paso de las cátedras a los programas transversales e institucionalmente llevados a la práctica; también urge que los esfuerzos en este campo sean perdurables y obedezcan a un genuino interés pedagógico e investigativo por parte de los docentes. Esto podría redundar en la promoción de una cultura académica más sólida y productiva que lleve la investigación a un campo más propositivo (pp. 200-201).

De una manera general, es posible pensar que hay algunas tendencias teóricas a destacar que subyacen a las experiencias, sin que estas sean, en sentido estricto, predominantes. Varias propuestas se consideran constructivistas y socioculturalistas. Se preguntan, particularmente, por el sentido y significado de los textos, por las dificultades para la enseñanzaaprendizaje y por las implicaciones para la interrelación social y cognitiva que se les plantean a sus actores. Se preguntan también, desde las prácticas culturales, por las posibilidades y obstáculos en la comunicación y expresión, al igual que sobre la construcción y manejo de sentido, de intenciones y propósitos en circuitos sociales específicos. Otra tendencia, en cambio, consideraría esencialmente las posibilidades cognitivas que se desprenden del desarrollo de la cultura letrada, así como las posibilidades de ampliación de las fronteras de conocimiento y de pensamiento. Por último, una tercera tendencia se centra en la comprensión de las estrategias utilizadas para escribir y leer, en sus procesos y metacogniciones.

Más que escuelas o modelos, además del constructivismo ya mencionado y, por ende, de Vigotsky, más bien se citan autores, mostrando influencias y procesos que posiblemente apenas estén empezando a asentarse; además, por supuesto, de una transformación radical en la consideración de la lectura y la escritura en la universidad colombiana. Ya no se piensa en la escritura como un campo estático en el que importa considerar básicamente los resultados, la calidad de los textos, la redacción precisa y la gramática; hay otros factores a considerar como la comunicación, los 
medios utilizados o la motivación. La escritura y la lectura no solo construyen conocimientos, también desarrollan y amplían la subjetividad y las habilidades de pensamiento de sus actores; la escritura y la lectura se realizan en medio de interacciones sociales que inciden en los mismos procesos de enseñanza-aprendizaje.

En cuanto a las estrategias didácticas, se comprenden como las acciones pedagógicas intencionadas que favorecen el logro del objetivo establecido por cada una de ellas, "un sistema de planificación aplicado a un conjunto articulado de acciones, que permite conseguir un objetivo y sirve para obtener determinados resultados" (Velasco \& Mosquera, s.f., p. 2).

En este sentido y al analizar las experiencias descritas, este aspecto es presentado desde dos posibilidades: la primera, la estrategia didáctica como la forma en que se enseñan o estructuran las experiencias, ligada a la metodología o al conjunto de estrategias e instrumentos para hacerla posible; y la segunda, una descripción de las estrategias para reflexionar sobre la enseñanza (Camilloni, Cols, Basabe \& Feeney, 2007).

Por la forma en que se presenta la información, la mayoría de las experiencias está ubicada en la primera posibilidad y su despliegue permite ver los caminos metodológicos que llevan a la interacción de los estudiantes con las teorías o prescripciones y las prácticas. Las experiencias denotan la comprensión de lectura y escritura como habilidades que se potencian en su ejercicio, por ello, el taller es tan recurrente en este aspecto; es decir, aprender haciendo.

Estas estrategias convergen en la descripción de procedimientos entre los que imperan, en la escritura de textos: la revisión, la re-escritura y la puesta en escena del escrito. En lectura, los procedimientos son (sin que signifique que en todos los casos todas se llevan a cabo): lectura de textos, discusión, escritura reflejo de la comprensión lectora, del posicionamiento frente a la lectura y/o punto de vista. La presentación de estas estrategias posibilita la planeación y el desarrollo de formas de trabajo en el aula como las asesorías en procesos individuales o en grupo, la corrección entre pares, la lectura en voz alta.

El aporte didáctico de las estrategias es limitado; no se cuestiona la relación de la lectura y la escritura con la cultura académica, con la infraestructura curricular y con la identidad profesional de los estudiantes, pues no es reflexiva en los procesos de enseñanza y eso dificulta que se proyecte en los procesos de aprendizaje. Esto llama la atención, pues en la lectura y escritura el docente universitario podría encontrar los insumos para la consolidación de un saber didáctico disciplinar.

Sobre los recursos, hay diversidad de ellos a la hora de hacer la lectura y escritura. Las herramientas de conocimiento y de desarrollo de procesos de pensamiento pasan por el manejo de recursos audiovisuales, videojuegos, cuentos e imágenes, entre otras.

La tipología de texto no solo se justifica desde la diversidad de carreras profesionales en las que las experiencias se desarrollan, sino en las múltiples intenciones con las que se programan los cursos, talleres, electivas o pruebas. Se mencionan los textos expositivos, los informativos, los técnicos, los narrativos y los argumentativos. Sin embargo, no se menciona si los textos seleccionados guardan alguna relación con los tipos de textos que a diario leen los estudiantes en la carrera.

Muy pocas experiencias están ubicadas en la reflexión por la enseñanza, es decir, en la pregunta por el sujeto, su historia lectora y escritora, su referencia a la hora de entender estos procesos en la realidad o en contexto y la metacognición como camino para la reflexión. Algunas de las experiencias se acercan a este aspecto desde la formulación de estrategias que provocan la concientización de los estudiantes al reconocer la lectura y la escritura como prácticas socioculturales, en consonancia con Anne-Marie Chartier (2004), o bien mediante algunas estrategias metacognitivas con las que se acercan a la reflexión sobre sus propias necesidades.

Algunas experiencias presentan estrategias que responden a procesos que dan sentido a la vida académica en la universidad como socialización de escritos, defensa pública de los textos, sistematización y recolección de información alrededor de lo que se entiende y se vive como lectura y escritura. En este aspecto es interesante que varias de las experiencias se han pensado desde la investigación, con una definición clara de los tipos de estudio, las metodologías y la presentación de resultados.

Por otra parte, llama la atención la relación existente entre las estrategias didácticas y la concepción del currículo. Para este estudio, la idea de currículo está ligada a la de plan de estudio, a los niveles, a la enseñanza en la propuesta de formación de un programa; esto ayuda a identificar la necesidad que tienen las instituciones de educación superior de establecer espacios para la oralidad, la lectura y la escritura por su importancia, su incidencia en la formación de los profesionales y muchas veces por convertirse en un requisito en la culminación de los estudios, aunque algunas experiencias tienen origen en la iniciativa de docentes o estudiantes o en productos de investigación.

Como se ha reiterado, las propuestas estudiadas se desarrollan como espacios únicos, que tienen poca o nula interacción con las asignaturas propias de las carreras de los estudiantes. Así mismo, dado el carácter de los objetivos, las estrategias están pensadas para reforzar o afianzar en los estudiantes estrategias para la comprensión y la producción de textos. Esto 
explica la tendencia al taller, que de manera expresa se ofrece en doce (12) de las propuestas.

Este aislamiento de los espacios de las asignaturas impide que el aprendizaje de los estudiantes, reflejado en la transferencia de saberes hacia otros espacios académicos, se vea imposibilitado. En estudios recientes que analizan las prácticas llevadas a cabo en las asignaturas en las que no se afianzan como objetivo los procesos de lectura y escritura (González \& Vega, 2010; Pérez et al., 2010), se observa cómo los estudiantes no utilizan las estrategias que les ofrecieron en los cursos de primer año, pues las exigencias son otras y hasta las formas de denominar los subprocesos son otras, con lo cual el estudiante no establece la relación entre estas prácticas y las que les exigen en las materias. Se podría decir que se privilegian estrategias artificiales de lectura y escritura que pretenden afianzarlas para la educación superior, pero desde un enfoque asignaturista, en el que poco o nada intervienen los profesores de las demás materias y en las que las propuestas de textos y necesidades de lectura para la academia están invisibilizadas. Una forma de minimizar estas prácticas desconectadas de las realidades académicas de los estudiantes, es el hecho de que en varias de ellas (10) se hacen expresos la socialización de los textos y el carácter colaborativo en ellas.

En estas propuestas, el acompañamiento personalizado que requieren los procesos de lectura y escritura y la necesidad de orientar al estudiante para apropiarse del lenguaje de una disciplina para que logre comunicarse en ese lenguaje es escaso (solo dos de ellas ofrecen acompañamiento personalizado). Sobre este particular, varios autores -entre ellos, Paula Carlino (2005) - han insistido en que solo se lograría con el apoyo de un especialista.

Un último aspecto por resaltar y que ya advertía Albert R. Kitzhaber (citado por Russell, 1995) al hacer referencia a los cursos de lenguaje que se ofrecieron en Estados Unidos desde finales del siglo XIX, es la poca correspondencia entre la cantidad de temas por trabajar, los ingentes esfuerzos de los docentes y los resultados visibles al final de los cursos de lectura y escritura de primer año ofrecidos por las universidades que, para nuestro caso colombiano, no duran más de 15 semanas. Son muchos los temas que se quieren abordar en estos cursos, a juzgar por los testimonios expuestos por los informantes (comprensión, interpretación, argumentación, escritura con propósitos específicos, elaboración de borradores, aspectos de investigación, aspectos formales de lengua, pensamiento crítico, pensamiento lógico, etc.); esto hace que a cada tema se le inviertan solo unas pocas horas. En este panorama, el aprendizaje significativo es solo una buena intención.

Una vez más, los datos arrojados constatan el estado incipiente aún de la discusión del tema, a pesar de los más de diez encuentros nacionales e internacionales que se cuentan en el país, adelantados por diferentes universidades de diversas regiones. De las investigaciones a la práctica no siempre hay un camino recto y llano; que se conviertan en propuestas sólidas y sostenidas en el tiempo requiere aún mucha conciencia por parte de las instituciones. Abundan los esfuerzos, en grupo y en solitario, por parte de muchos docentes. A la fecha, hay más de ochenta programas de lectura y escritura, pero sin mayor incidencia en las instituciones (González \& Vega, 2010). La despreocupación por parte de muchos docentes que aún no encuentran el poder epistémico de la lectura y la escritura pues las abandonan a un solo espacio académico en la mayoría de los casos, reduce las posibilidades de posicionar estos procesos como centrales y prioritarios en las instituciones. 


\section{Conclusiones y recomendaciones}

Además de la reflexión que ya se ha realizado, lo más valioso del estudio es que permite hacer historia de cómo se ha empezado y se ha venido dando la discusión en lectura y escritura en la universidad. Además, de ser un interés visible en el país, se nota apertura por cualificar y socializar las experiencias que tienen qué ver con este tema, aunque implique grandes retos como insistir en la necesidad de avanzar en la investigación en Colombia; retomar dificultades de la indagación; confirmar los objetivos; contrastar estos hallazgos con los de otras investigaciones; resaltar el largo camino por recorrer para lograr políticas institucionales; reconocer la inmadurez en la que estamos para hablar de lectura y escritura en las disciplinas y en la cultura académica.

Este estudio ha permitido revelar las implicaciones que trae pensar la lectura y la escritura como componentes ajenos a la reflexión sobre la formación profesional y sobre la enseñanza. Cada una de las experiencias se presenta de manera aislada a una concepción o postura de lectura y escritura en la cultura académica de la educación superior, pues su planeación está sujeta a las decisiones del docente que programa la asignatura, taller, prueba o curso. Indudablemente, los procesos de formación en lectura y escritura pasan por los sujetos, pero cuando se piensa en una cultura académica no se quedan solo en ellos, pues sobrepasa la experiencia particular y se construye desde diferentes miradas como las políticas, la trayectoria, los propósitos de formación, las tradiciones y los escenarios de desarrollo, entre otras.

Desde esta perspectiva, las experiencias en lectura y escritura pueden llegar a transformar a los docentes y estudiantes pero no a la cultura, debido a que su formulación está definida en la inmediatez de un plan de estudios, de un currículo, de un programa de selección o de una propuesta de capacitación, lo que infortunadamente aún impera.

Pero, ¿cómo lograr incidir en la cultura? No podemos pensar que es un proceso directivo, instruccional, obligatorio; por el contrario, se debe pensar como un proceso reflexivo de discusión y sobre todo de permanente desarrollo, que se puede ir empoderando en el ambiente por medio de las prácticas, de algunas propuestas articuladas a propósitos institucionales en el marco de la formación y la profesión, de las representaciones socialmente construidas que identifican los saberes de la institución y de las tradiciones, entre otros elementos. Por lo tanto, no resulta fácil de medir o de planear a corto plazo, se trata entonces de hacerlo consciente en los

sujetos y visualizarlo a largo plazo.

Tal vez por esto las instituciones de educación superior piensan erradamente en la lectura y la escritura como habilidades y destrezas del lenguaje, no como prácticas sociales y culturales que hacen parte de la cultura académica y que "dan significado a las acciones que fortalecen las relaciones intersubjetivas, disciplinares, científicas y si se analizan desde el sentido y el valor que le adjudican las personas a estas acciones, pueden convertirse en prácticas sociales que dinamizan y le dan identidad a dicha cultura" (Sevilla \& Blandón, 2010).

Visto de esta manera, la experiencia de enseñanza de la lectura y escritura en la universidad toma sentido desde la cultura y no desde las necesidades de la academia, aunque los sujetos no las sienten como propiedades adquiridas, sino que las comprenden como prácticas que les permiten interactuar con el conocimiento, con el ambiente académico, con la construcción de su ser profesional y con la relación con otros. 
Las experiencias son susceptibles de ser replicadas en otra institución pero en ningún caso se manifiesta una acción que conduzca a que esto sea realidad. De nuevo, los esfuerzos aislados prevalecen, aunque algunos están de acuerdo en que los encuentros, simposios y congresos organizados por la REDLEES han contribuido hasta el momento a vislumbrar esta transferencia.

En cuanto a la financiación, las experiencias han sido y son asumidas por las instituciones con cargo al presupuesto y nómina de los programas académicos y/o facultades; vale decir que solo una experiencia cuenta con financiamiento externo del Departamento Administrativo de Ciencia y Tecnología e Innovación de Colombia (Colciencias). Este aspecto debería replantearse; según Charles Bazerman³ (2009), considerar la financiación externa mediante convenios empresariales puede ser una alternativa.

Carlos Enrique Mendoza-Arciniegas es publicista egresado de la Universidad de Bogotá Jorge Tadeo-Lozano. Con posgrado en docencia universitaria, investigador académico y docente universitario. Ha sido creativo publicitario, planner estratégico, empresario y administrador educativo en varias universidades del país. Decano de facultad, director de programa, director de desarrollo académico, coordinador de humanidades, coordinador de acreditación.

Alberto Echeverri-Guzmán es doctor en teología, Universidad Pontificia Gregoriana, Roma. Licenciado en filosofía y letras con énfasis en filosofía, Pontificia Universidad Javeriana, Bogotá. Estudios postdoctorales en ciencias sociales, Universidad Pedagógica Nacional, Bogotá. Miembro de la Red de Lectura y Escritura en Educación Superior, REDLEES, desde 2009.

Daniel Quecán-Castellanos es licenciado en español e inglés, Universidad Pedagógica Nacional con vinculación de 18 años de labores con la Universidad de Ciencias Aplicadas y Ambientales, UDCA, con 4 años de experiencia en investigaciones afines a la lectura y escritura en educación superior. Miembro de la Red de Lectura y Escritura en Educación Superior, REDLEES, desde 2009.

Luis Ernesto Pardo-Rodríguez es bibliotecólogo y archivista, Universidad de La Salle. Especialista en pedagogía de la comunicación, Universidad Distrital. Magíster en docencia, Universidad de La Salle. Profesor del programa de sistemas de información y documentación, Universidad de La Salle y profesor de la Facultad de Comunicación Social para la Paz, Universidad Santo Tomás.

3 Reflexión hecha por el autor en un conversatorio de ASCUN, realizado en Bogotá en 2009, para mostrar resultados parciales del estudio. 
María Floralba Angulo-Abaunza es licenciada en lenguas modernas, Universidad de La Salle. Magíster en lingüística aplicada a la enseñanza del inglés como lengua extranjera, Universidad Distrital Francisco José de Caldas. Docente investigadora, Universidad de La Salle. Línea de investigación: lenguaje, cultura y didácticas de las lenguas.

Juan Manuel Silva-García es filósofo, Universidad Nacional. Magíster en literatura, Pontificia Universidad Javeriana. Docente de tiempo completo, Universidad Militar Nueva Granada, Facultad de Relaciones Internacionales, Estrategia y Seguridad. Maestría en Relaciones y Negocios Internacionales. Escritor e investigador.

Mariano Lozano-Ramírez es magíster en lingüística, abogado y especialista en pedagogía y docencia universitaria. Profesor investigador de la UniSabana. Exinvestigador titular del Instituto Caro y Cuervo. Investigador del grupo Valor y Palabra (UniSabana). Coinvestigador de la Comisión de Lingüística de la Academia Colombiana. Coordinador del Nodo Bogotá-Centro (REDLEES). Miembro de la Asociación de Lingüística y Filología de América Latina (ALFAL) y del comité de investigación de REDLEES.

\section{Bibliografía}

Acevedo, J. (2009). Conocimiento didáctico de contenido para la enseñanza de la Naturaleza de la Ciencia. Revista Eureka sobre Enseñanza y Divulgación de las Ciencias, 6 (1), 21-46. Disponible en: http://www. todosaaprender.edu.co/articles-348167 conocimiento ciencia.pdf

Bazerman, C. (1988). Shaping Written Knowledge. The Genre and Activity of the Experimental Article in Science. Wisconsin: The University of Wisconsin Press. Disponible en: http://wac.colostate.edu/books/ bazerman_shaping/shaping.pdf

Bazerman, C. (2009). Conversatorio sobre experiencias de lectura y escritura en Colombia. Bogotá.

Bazerman, C.; Little, J.; Chavkin, T.; Fouquette, D.; Bethel, L. \& Garufis, J. (2005). Writing across the Curriculum. Reference Guides to Rhetoric and Composition. West Lafayette, Indiana: Parlor Press LLC. Disponible en: http://wac.colostate.edu/books/bazerman_wac/wac.pdf

Bazerman, C. \& Prior, P. (2005). Participating in Emergent Socioliterate Worlds. Genre, Disciplinarity, Interdisciplinarity. En Richard Beach, Judith Green, Michael Kamil \& Timothy Shanahan. Multidisciplinary Perspectives on Literacy Research, 133-178. 2 ed., Nueva Jersey: Hampton Press Inc. Disponible en: http://mina.education.ucsb.edu/ bazerman/chapters/documents/Bazerman2005ChpterSocioliterate Worlds.pdf

Bazerman, C. \& Russell, D. (eds.) (1995). Landmark Essays on Writing across the Curriculum. New York: Routledge.

Camargo, Z.; Uribe, G.; Caro, M. \& Castrillón, C., (2009). Estado del arte de las concepciones sobre prácticas de lectura y escritura en la universidad colombiana. Artículo insumo del proyecto de investigación Análisis de una muestra representativa de los relatos presentados al Concurso Nacional de Cuento Gabriel García Márquez (2007), adelantado por el Grupo de Investigación en Didáctica de la Lengua Materna y la Literatura (DiLeMa), por convenio entre el Ministerio de Educación Nacional y la Universidad del Quindío (2008). Disponible en: http://186.113.12.12/discoext/collections/ 0023/0045/02590045.pdf

Camilloni, A.; Cols, E.; Basabe, L. \& Feeney, S. (2007). El saber didáctico. Buenos Aires: Paidós. 
Carlino, P. (2005). Escribir, leer y aprender en la universidad. Una introducción a la alfabetización académica. Buenos Aires: Fondo de Cultura Económica, FCE.

Carlino, P. (2009). Prácticas y representaciones de la escritura en la universidad los casos de Australia, Canadá, EE.UU. y Argentina. Cuaderno de Pedagogía Universitaria, 6 (12), 6-17. Pontificia Universidad Católica Madre y Maestra, Santiago, República Dominicana. Disponible en: http://www.pucmm.edu.do/cdp/Documents/CuadernodePedagogia No12.pdf

Chartier, A. M. (2004). Enseñar a leer y escribir. Una aproximación histórica. México: Fondo de Cultura Económica, FCE.

Cisneros, M. \& Vega, V. (2011). En busca de la calidad educativa a partir de los procesos de lectura y escritura. Pereira: Universidad Tecnológica de Pereira, UTP.

Colombia (1994). Ley 115 de 1994, por la cual se expide la Ley General de Educación. Diario Oficial, 41.214, 8 de febrero de 1994. Disponible en: http://www.alcaldiabogota.gov.co/sisjur/normas/Norma1. jsp? $\mathrm{i}=292$

Ferreiro, E. (2002). Pasado y presente de los verbos leer y escribir. Buenos Aires: Fondo de Cultura Económica, FCE.

González, B. (2009). Strategies, Policies and Research on Reading and Writing in Colombian Universities. En Charles Bazerman, Robert Krut, Karen Lunsford, Susan McLeod, Suzie Null, Paul Rogers \& Amanda Stansell (eds.). Traditions of Writing Research, 122-132. New York, Londres: Routledge.

González, B. \& Salazar-Sierra, A. (eds.) (2015). Formación inicial en lectura y escritura en la universidad. De la educación media al desempeño académico en la educación superior. Bogotá: Pontificia Universidad Javeriana.

González, B. \& Vega, V. (2010). Prácticas de lectura y escritura en la universidad. El caso de cinco asignaturas de la Universidad Sergio Arboleda. Bogotá: Fondo de Publicaciones de la Universidad Sergio Arboleda. Disponible en: http://cedoc.infd.edu.ar/upload/PracticasLectura Escritura.pdf

González, B. \& Vega V. (2012). Lectura y escritura en la educación superior colombiana: herencia y deconstrucción. Revista Interacción, 12, 195201. Disponible en: http://www.unilibre.edu.co/revistainteraccion/ volumen12/art13.pdf

Greene, S. \& Lidinsky, A. (2008). From Inquiry to Academic Writing: A Text and Reader. Boston: Bedford/St. Martin's.

Mendoza, C. et al. (2011). ¿Qué dicen las instituciones de Educación Superior en torno a la Lectura y la Escritura? Informe de la investigación interinstitucional, llevada a cabo por el comité de investigación del nodo Bogotá de REDLEES. Texto inédito.

Ministerio de Educación Nacional, MEN (2006). Guía metodológica para usuarios. Banco de experiencias significativas en educación superior (en línea). Disponible en: http://www.colombiaaprende.edu.co/html/ directivos/1598/articles-102621_Archivo1.doc

Ortiz, E. (2011). La escritura académica universitaria: estado del arte. Ikala. Revista de Lenguaje y Cultura, 16 (28), 17-41. Disponible en: http:// aprendeenlinea.udea.edu.co/revistas/index.php/ikala/article/view File/7815/9106

Peña, L. (2009). La competencia oral y escrita en la educación superior. Documento inédito que hace parte del trabajo realizado por el autor en el marco del Comité Consultivo para la Definición de Estándares 
y Evaluación de Competencias Básicas en la Educación Superior, conformado por iniciativa del Ministerio de Educación Nacional. Versión de 2008 disponible en: http://www.mineducacion.gov.co/1621/ articles-189357_archivo_pdf_comunicacion.pdf

Pérez, M. et al. (2010). ¿Para qué se lee y se escribe en la universidad colombiana? Un aporte a la consolidación de la cultura académica. Coloquio realizado en la Universidad Javeriana de Bogotá, en el marco de la discusión y socialización de resultados de investigación del estudio que lleva su nombre.

Pérez, M. \& Rincón, G. (coords.) (2013). ¿Para qué se lee y se escribe en la universidad colombiana? Un aporte a la consolidación de la cultura académica del país. Bogotá: Pontificia Universidad Javeriana. Disponible en: http://wac.colostate.edu/books/colombian/highered.pdf

Red de Lectura y Escritura en Educación Superior, REDLEES (2007). Reglamento de REDLEES.

Red de Lectura y Escritura en Educación Superior, REDLEES (2014). Reflexiones y propuestas para pensar una política institucional de desarrollo de la lectura y la escritura en Educación Superior.

Russell, D. (1995). Activity Theory and its Implications for Writing Instruction. En Joseph Petraglia (ed.). Reconceiving Writing, Rethinking Writing Instruction, 51-78. Hillsdale, New Jersey: Erlbaum. Disponible en: http:// www. public.iastate.edu/ drrussel/papers/AT\%20Implications\%20 (Pet\%20II).doc

Sevilla, O. \& Blandón, F. (2010). Reflexiones del docente universitario sobre la producción de textos académicos. Ponencia en el IV Encuentro Nacional y III Internacional Lectura y Escritura en Educación Superior. Santa Marta, Colombia. Disponible en: https://campusvirtual.univalle. edu.co/moodle/mod/resource/view.php?id=123510

Uribe, G. \& Camargo, Z. (2011). Prácticas de lectura y escritura académicas en la universidad colombiana. magis, Revista Internacional de Investigación en Educación, 3 (6), 317-341. Disponible en: http://dialnet. unirioja.es/descarga/articulo/4434038.pdf

Velasco, M. \& Mosquera, F. (s.f.). Estrategias didácticas para el aprendizaje cooperativo. Disponible en: http://acreditacion.udistrital.edu.co flexibilidad/estrategias_didacticas_aprendizaje_colaborativo.pdf 